\title{
Lasing Characteristics of 12-Fold Symmetric Quasi-periodic Photonic Crystal Slab Nanolasers
}

\author{
Kengo NOZAKI and Toshihiko BABA \\ Department of Electrical and Computer Engineering, Yokohama National University, 79-5 Tokiwadai, \\ Hodogaya-ku, Yokohama 240-8501, Japan
}

We fabricated a 12-fold symmetric quasiperiodic photonic crystal (QPC) into a GaInAsP slab, and obtained a lasing action in a single-defect nanocavity and a defect-free area at room temperature. Four defect modes observed by a lithographic tuning were clearly explained by finite-difference time-domain calculation. The lowest threshold was obtained for the quadrupole mode, which has a small modal volume of 0.58 times the cubic wavelength in the cavity, by the fine-tuning of adjacent airholes to the defect. The lasing in the defect-free area was also suggested to be due to the localization of two-dimensional Bragg modes at the photonic gap edge. Because of its high structural flexibility, the QPC nanocavity has the potential of becoming a good alternative to the PC nanocavity.

Photonic crystal, Quasiperiodic photonic crystal, Nanocavity, Nanolaser, GaInAsP, Localized mode, Bragg mode, Purcell effect

\section{Introduction}

The photonic crystal (PC) has a periodic structure with a translational symmetry and exhibits a photonic band gap (PBG). It enables us to form a nanocavity with an ultrasmall modal volume and ultrahigh $Q$ by only introducing a point defect. ${ }^{1-4)}$ Such a nanocavity in an emitting material such as III-V semiconductors achieves efficient electron-photon interaction, i.e., enhancement in spontaneous emission rate (Purcell effect) under the weak-coupling regime and vacuum Rabi splitting under the strong-coupling regime. ${ }^{4-7)}$ Even without point defects, lasing also occurs in the PC due to the distributed feedback of light at a photonic band edge. ${ }^{8-11)}$ On the other hand, the quasiperiodic photonic crystal (QPC) does not have a translational symmetry but high-order rotational and line symmetries (self-symmetry) ${ }^{12-14)}$ It does not maintain Bloch waves, as in PCs, so the photonic band and Brillouin zone cannot be defined. However, Bragg conditions can be defined by reciprocal points, and photonic gaps such as PBGs occur. Therefore, lasing operation with a variety of modes is expected. ${ }^{15)}$

In our previous study, we observed the lasing of a whispering gallery mode in a seven-hole-missing defect fabricated into a 12-fold symmetric QPC slab, and also observed that of a two-dimensional (2-D) Bragg diffraction mode in its defect-free area. ${ }^{16)}$ Recently, Lee et al. have reported lasing in a single-defect cavity in a similar QPC slab. ${ }^{17)}$ However, they limited their design to a relatively small lattice constant ranging from 350 to $400 \mathrm{~nm}$ to focus on the lasing action by the hexapole mode, although such small lattice constants are difficult to fabricate. In this study, we show that not only the hexapole mode but also various localized and Bragg modes appear as lasing modes for larger lattice constants.

In the next section, we explain the fabrication process of the device. Then, in $\S 3$, we present the lasing characteristics of the single-defect nanolaser and its spectral shift by lithographic tuning. In $\S 4$, we show the frequency characteristics of defect modes, and the correspondence between the experiment and finite-difference time-domain (FDTD) calculation. We also discuss the threshold characteristics that are dependent on some structural parameters. In $\S 5$, we discuss the frequency characteristics and the modal fields of the Bragg modes observed in the defect-free area.

\section{Device Fabrication}

We prepared a $1.55 \mu \mathrm{m}$ GaInAsP/InP epitaxial wafer with a slab active layer consisting of five quaternary compressively strained quantum wells (CS-QWs) and separate-confinement heterostructure layers. In addition to the CS-QWs, the active layer included $1.2 \mu \mathrm{m}$ quaternary (Q) barrier layers of $10 \mathrm{~nm}$ thickness, and 1.2, 1.15, and $1.1 \mathrm{Q}$ separate-confinement heterostructure layers, each of which was $30 \mathrm{~nm}$ in thickness. Thus, the total thickness of the active layer was $240 \mathrm{~nm}$. Airholes in the QPC 
lattice were formed in the slab by e-beam lithography and HI/Xe inductively coupled plasma (ICP) etching, ${ }^{18,19)}$ and the airbridge structure was formed by $\mathrm{HCl}$ selective wet etching. Because the $\mathrm{HI}$ gas allows etching at $\sim 70^{\circ} \mathrm{C}, e$-beam resist can be directly used as an etching mask. It simplifies and improves the PC pattern, compared with the metal or dielectric mask process. In the ICP etching using RIE-200ip (Samco), we set the HI/Xe flow rates, gas pressure, ICP power and bias power to be 0.2/0.1 sccm, $0.28 \mathrm{~Pa}, 100 \mathrm{~W}$, and $300 \mathrm{~W}$, respectively. Figure 1 shows the top view of the fabricated device and the lattice structure of the 12-fold symmetric QPC composed of tiled square and equilateral triangular unit cells based on the Stampfli inflation rule. For the single-defect nanocavity, the lattice constant $a$ and the normalized airhole diameter $2 r / a$ were designed to be $480-600 \mathrm{~nm}$ and $0.50-0.75$, respectively. These parameters generate a photonic gap at $\lambda \sim 1.55 \mu \mathrm{m}$ for the in-plane polarization. Also, the radius of the innermost airholes $r^{\prime}$ was reduced to $0.7 r-1.0 r$ to improve the modal $Q$ factor. Defect-free devices were also fabricated with $a=440-580 \mathrm{~nm}$ and $2 r / a=0.53-0.67$.

\section{Defect-Mode Lasing}

For the measurement, each device was photopumped at room temperature by $0.98 \mu \mathrm{m}$ pulsed laser light with a duty ratio of $0.075 \%$ and a focused spot diameter of $\sim 3.5 \mu \mathrm{m}$. Emitted light was detected by a multimode fiber whose tip was placed $\sim 100 \mu \mathrm{m}$ from the device and inclined $30^{\circ}$ to the substrate plane, and then analyzed by an optical spectrum analyzer. The lasing action was observed in many samples of the single-defect nanocavity with $a=480-600 \mathrm{~nm}$, when the pump light was overlapped with the defect. Figure 2(a) shows the lasing characteristics showing the lowest threshold. Due to the restriction of the measurement system used, it was difficult to clearly evaluate the subthreshold data. However, the lasing was confirmed from the clear single-mode spectrum above the threshold. The threshold pump power was estimated to be $0.5 \mathrm{~mW}$ from the above-threshold slope. This value is almost the same as that for a single-defect nanocavity in a triangular-lattice PC fabricated and measured in the same way. The lasing frequency shifts as $2 r^{\prime} / a$ changes. Figure 2(b) shows the spectral shift due to wider lithographic tuning of $a$ and $2 r / a$. As $2 r / a$ increases against the constant $a$, the cavity length decreases and hence, the mode peak blue-shifts. Although each spectrum shows single-mode lasing, the continuity of the spectral shift reveals that four different lasing modes appear in the lithographic tuning.

Figure 3(a) shows a summary of the normalized frequency $a / \lambda$ of four different modes in the single-defect nanocavity as a function of $2 r^{\prime} / a$. The frequencies of the four modes are clearly separated, and linearly blue-shifted with $2 r^{\prime} / a$. In order to specify these modes, we performed the 3-D FDTD calculation, in which the cavity structure observed by high-resolution scanning electron microscopy was precisely modeled. Here, a cubic Yee cell of side $20 \mathrm{~nm}$, a slab index $n$ of 3.4 and $\sim 13$ rows of airholes around the cavity were assumed. The calculated results are shown by thick dashed lines in Fig. 3(a). They are in good agreement with the experimental plots for four modes. Figure 3(b) shows the $\left|H_{z}\right|^{2}$ distribution (square magnetic field normal to the slab), normalized frequency $a / \lambda, Q$ factor and mode volume $V_{\mathrm{m}}$, which were calculated for $2 r / a=0.70$ and $2 r^{\prime} / a=0.55$. They are the quadrupole mode, Bragg-like modes 1 and 2, and dipole mode, respectively. The quadrupole and dipole modes are almost confined inside the defect. On the other hand, the Bragg-like modes 1 and 2 are localized outside of the defect. We consider that the localization is caused by the offspring lattice of the QPC, as shown by the thick solid line in Fig. 1(b). Similar discussion can be made for the parent lattice later.

The calculated $Q$ factors of these modes were mainly determined by the out-of-plane loss to be $400-1300$. We think that such low values were caused by the calculation model. Because the QPC model has no translational symmetry, its lattice constant can be disordered by the Yee cell of the FDTD calculation, which results in excess scattering loss. In addition, the total size of the model is limited, whereas the field penetration depth into the QPC is twice as long as that into the PC. Therefore, the field can reach the edge of the QPC slab, resulting in additional scattering loss. In the experiment, the $Q$ factor may be higher, because the disordering in the lattice constant is smaller and the device size is threefold larger than the calculation model. The mode volume $V_{\mathrm{m}}$ was calculated using the definition shown in ref. 1 . In contrast to the large $V_{\mathrm{m}}$ of the Bragg-like modes, the quadrupole mode and the dipole mode have very small $V_{\mathrm{m}}$, which is almost the same as that in a triangular-lattice PC single-defect nanocavity, even though with the long penetration depth of the field. 


\section{Threshold Characteristics}

Figure 4(a) shows a summary of the thresholds of the four modes as functions of $2 r^{\prime} / a$ for single-defect cavities. The grouping circle for each mode indicates that the threshold for the quadrupole mode is low overall, and that the lowest value of $0.5 \mathrm{~mW}$ was obtained by this mode. Furthermore, the effective power contributing to the lasing of this mode is lower than this value and much lower that for the Bragg-like modes, because the overlap efficiency of the quadrupole mode with the pump light is estimated to be only $\sim 20 \%$ due to the small modal volume. This result is qualitatively in agreement with the theoretical $Q$ factor shown in Fig. 3(b). Also, it is observed for all modes that lower threshold values are obtained for smaller $2 r^{\prime} / a$. One possible explanation for this is the structural dependence of the $Q$ factor. We performed the 3-D FDTD calculation on this dependence and noticed that the $Q$ factor takes its maximum value at $2 r^{\prime} / a \leq 0.4$. This is in good agreement with the result shown in Fig. 4(a). However, we cannot simply conclude that the low threshold values are only attributed to the improvement of the $Q$ factor. If the $Q$ factor is sufficiently high, then it will not be a crucial factor in determining the threshold, because other optical losses caused by the absorption and disordering become dominant. Also, carrier loss by the diffusion and surface recombination are other factors that mask the effect of the values of the $Q$ factor. ${ }^{20)}$ A smaller $2 r^{\prime} / a$ is also effective for reducing the surface recombination around the cavity. Therefore, this also explains the result shown in Fig. 4(a).

Figure 4(b) shows a summary of the thresholds of the quadrupole mode and the dipole mode as functions of lasing wavelength. Regardless of the gain spectrum peak of the wafer at $\lambda=1.55 \mu \mathrm{m}$, both modes show lower thresholds at longer wavelengths. We consider that this is caused by the strain relaxation effect in compressively strained quantum wells of the QPC slab, which results in the redshift of the gain spectrum. ${ }^{21)}$ In addition, the built-in potential by the strain relaxation can accelerate the carrier flow from the defect center to the airhole edge and enhance the surface recombination. This further supports the explanation of the lower threshold by the smaller $2 r^{\prime} / a$, because it suppresses strain relaxation and surface recombination.

\section{Defect-free Bragg Mode Lasing}

Similar lasing was observed for defect-free devices using the same photopumping. One unique characteristic we observed is that the lasing occurred whenever the pump light was altered by $7-8 \mu \mathrm{m}$. We consider that this is related to the degree of localization of the lasing mode and the overlap efficiency with the pump spot.

Figure 5(a) shows a summary of $a / \lambda$ for the lasing modes observed as functions of $2 r / a$. Here, three different modes were observed. Similar to the single-defect nanocavity, the frequencies of these modes are clearly separated and linearly blue-shifted with 2r/a. Gray areas shown in Fig. 5(a) indicate photonic gaps calculated by 3-D FDTD. All the modes were formed at the edge of the photonic gap, so they are 2-D Bragg diffraction modes. Figure 5(b) shows the time-averaged $\left|H_{z}\right|^{2}$ distributions and $a / \lambda$ calculated for $2 r / a=0.62$. The modes are extended over the model. They exhibit different symmetric patterns of Bragg modes reflecting their structure. For example, the antinode of Bragg mode 1 lies inside of the equilateral triangular lattice, whereas those of Bragg modes 2 and 3 are inside the square lattice. The slight nonuniformity of the patterns is caused by the limited size of the model; the phase condition of the field is slightly modified by the disordered reflection at the edge of the slab model. Even excluding this nonuniformity, the modes show some degree of localization. Similar to the discussion for Bragg-like modes 1 and 2 in the single-defect nanocavity, the localizations of these modes are considered to be prescribed by the parent lattice, as shown by the thick dashed line in Fig. 1(b). In the experiment, the size of the parent lattice was $7 \mu \mathrm{m}$ in diameter. It is a reasonable size which explains that the lasing was repeatedly observed by moving the pump light.

\section{Conclusion}

We fabricated a 12-fold symmetric QPC nanolaser into a GaInAsP quantum-well slab, and obtained a lasing action in the single-defect nanocavity and in the defect-free area at room temperature. The FDTD calculation explained the defect mode and Bragg-like mode in the single defect. The lowest threshold was $0.5 \mathrm{~mW}$ for the quadrupole mode, even though this mode has a relatively high normalized frequency of approximately 0.38 , at which the light cone circle is very large in the $k$ space. 
We also observed a threshold reduction by tuning the innermost airholes for the quadrupole mode and dipole mode. This might be due to the simultaneous suppression of the out-of-plane modal loss and the surface recombination. The lasing in the defect-free area was also explained by the localized 2-D Bragg mode at the photonic gap edge by the FDTD calculation. These results show that the lasing performance and the modal volume in the defect and the defect-free area are comparable to those of PC nanolasers. Therefore, the QPC nanocavity may be a good alternative to the PC nanocavity in future applications.

\section{Acknowledgement}

This work was supported by a Grant-in-Aid, the IT Program and the 21st COE Program from the Ministry of Education, Culture, Sports, Science and Technology, and a Grant-in-Aid and a research fellowship from the Japan Society of Applied Physics.

\section{References}

1) O. J. Painter, A. Husain, A. Scherer, J. D. O’Brien, I. Kim and P. D. Dapkus: J. Lightwave Technol. 17 (1999) 2082.

2) H. Y. Ryu, S. H. Kim, H. G. Park, J. K. Hwang, Y. H. Lee and J. S. Kim: Appl. Phys. Lett. 80 (2002) 3883.

3) H. Y. Ryu, M. Notomi, E. Kuramoti and T. Segawa: Appl. Phys. Lett. 84 (2004) 1067.

4) T. Baba, D. Sano, K. Nozaki, K. Inoshita, Y. Kuroki and F. Koyama: Appl. Phys. Lett. 85 (2004) 3989.

5) D. Englund, D. Fattal, E. Waks, G. Solomon, B. Zhang, T. Nakaoka, Y. Arakawa, Y. Yamamoto and J. Vučković: Phys. Rev. Lett. 95 (2005) 013904.

6) A. Kress, F. Hofbauer, N. Reinelt, M. Kaniber, H. J. Krenner, R. Meyer, G. Böhm and J. J. Finley: Phys. Rev. B 71 (2005) 241304.

7) T. Yoshie, A. Scherer, J. Hendrickson, G. Khitrova, H. M. Gibbs, G. Rupper, C. Ell, O. B. Shchekin and D. G. Deppe: Nature 432 (2004) 200.

8) M. Meier, A. Mekis, A. Dodabalapur, A. A. Timko, R. E. Slusher, J. D. Joannopoulos and O. Nalamasu: Appl. Phys. Lett. 74 (1999) 7.

9) M. Imada, S. Noda, A. Chutinan, T. Tokuda, M. Murata and G. Sasaki: Appl. Phys. Lett. 75 (1999) 316.

10) M. Notomi, H. Suzuki and T. Tamamura: Appl. Phys. Lett. 78 (2001) 1325.

11) H. Y. Ryu, S. H. Kwon, Y. J. Lee, Y. H. Lee and J. S. Kim: Appl. Phys. Lett. 80 (2002) 3476.

12) Y. S. Chan, C. T. Chan and Z. Y. Liu: Phys. Rev. Lett. 80 (1998) 956.

13) C. Jin, B. Cheng, B. Man, Z. Li, D. Zhang, S. Ban and B. Sun: Appl. Phys. Lett. 75 (1999) 1848.

14) M. E. Zoorob, M. D. B. Charlton, G. J. Parker, J. J. Baumberg and M. C. Netti: Nature 404 (2000) 740.

15) M. Notomi, H. Suzuki, T. Tamamura and K. Edagawa: Phys. Rev. Lett. 92 (2004) 123906.

16) K. Nozaki and T. Baba: Appl. Phys. Lett. 84 (2004) 4875.

17) S. K. Kim, J. H. Lee, S. H. Kim, I. K. Hwang, Y. H. Lee and S. B. Kim: Appl. Phys. Lett. 86 (2005) 031101.

18) T. Ide, J. Hashimoto, K. Nozaki, E. Mizuta and T. Baba: Jpn. J. Appl. Phys. 45 (2006) L102.

19) K. Nozaki, T. Ide, J. Hashimoto, W-H. Zheng and T. Baba: Electron. Lett. 41 (2005) 843.

20) K. Nozaki and T. Baba: IEEE J. Sel. Area Commun. 23 (2005) 1411.

21) M. Fujita, R. Ushigome and T. Baba: Appl. Phys. Lett. 80 (2002) 1511.

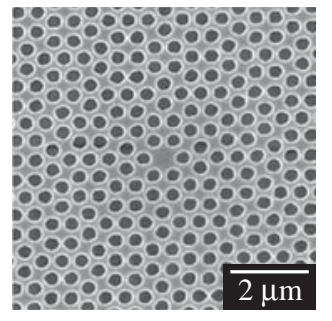

(a)

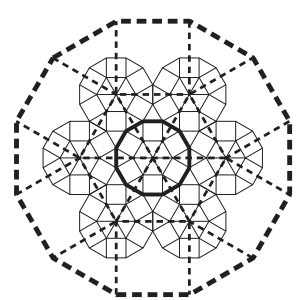

(b)

Fig. 1. 12-fold symmetric QPC structure. (a) Top-view of fabricated device. (b) Lattice structure. Dashed and solid lines show the parent lattice and offspring lattice, respectively. Thick lines for both lattices indicate the edge of these lattice units. 

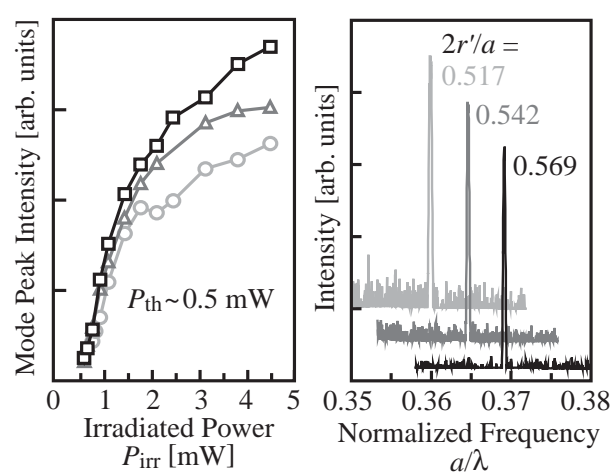

(a)

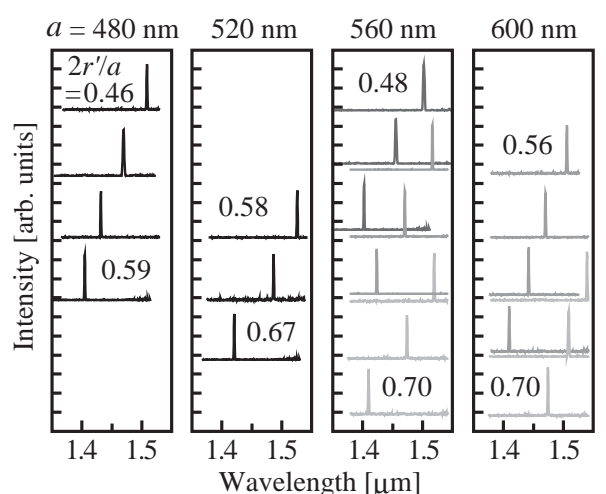

(b)

Fig. 2. Lasing characteristics observed by photopumping at room temperature in single-defect nanolaser. (a) Mode peak intensity vs irradiated pulse peak power characteristics and corresponding lasing spectra above threshold. The three different curves indicate the results for different values of the normalized airhole diameter $2 r^{\prime} / a$. (b) Lasing spectra observed for lithographic tuning of $a$ and $2 r^{\prime} / a$.

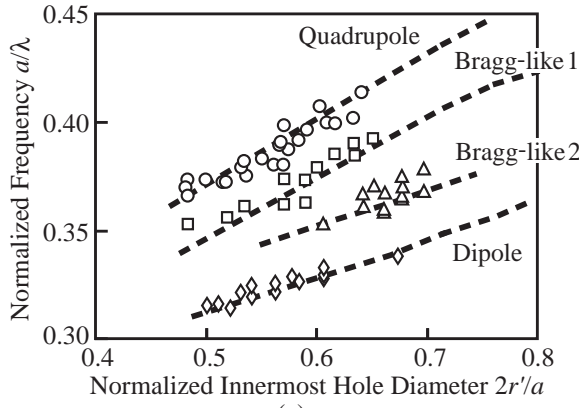

(a)

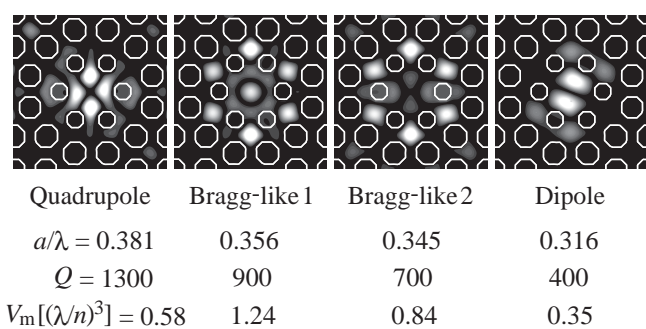

(b)

Fig. 3. Cavity modes in experiment and FDTD calculation for single-defect nanocavity. (a) Modal frequency shift vs $2 r^{\prime} / a$ characteristics. The plots show experimental results, whereas the dashed lines show calculated results. (b) $\left|H_{z}\right|^{2}$ distribution, normalized frequency $a / \lambda, Q$ factor and modal volume $V_{\mathrm{m}}$ of each mode calculated for $2 r / a=0.70$ and $2 r^{\prime} / a=0.55$.

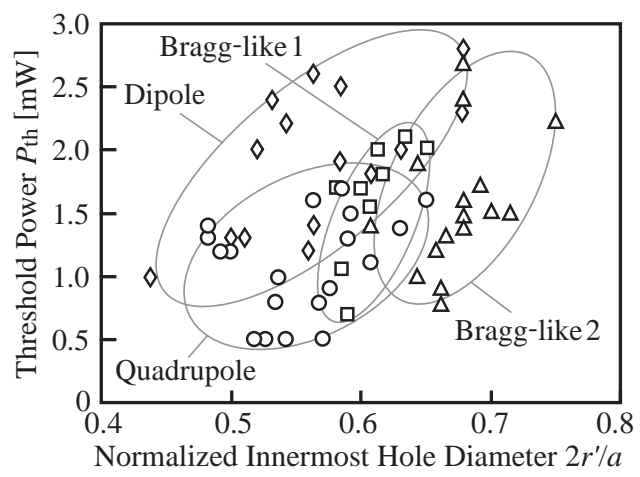

(a)

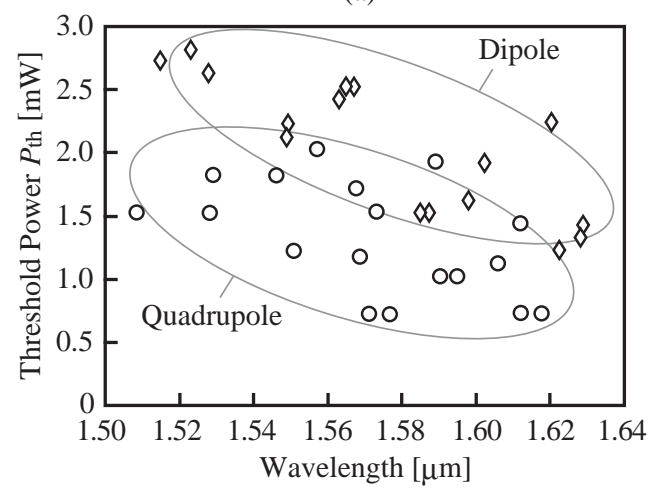

(b)

Fig. 4. Relations between $2 r^{\prime} / a$, lasing wavelength and threshold for single-defect nanolaser. (a) Threshold vs $2 r^{\prime} / a$ characteristics. (b) Threshold vs lasing wavelength characteristics. 


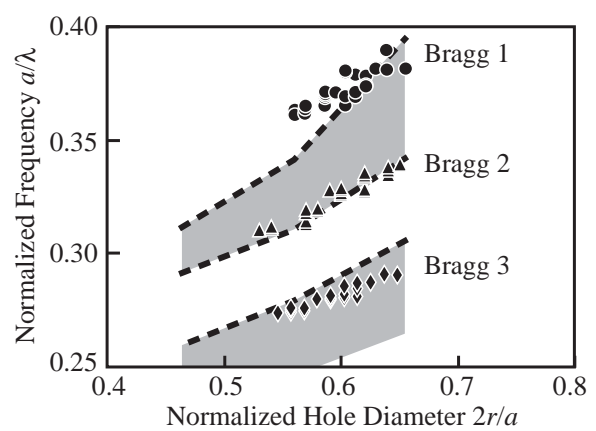

(a)

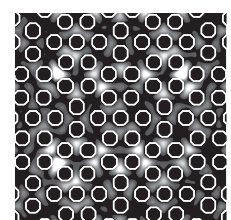

Bragg 1

Bragg 2

Bragg 3

$a / \lambda=0.363$

0.323

0.288

Fig. 5. Cavity modes in experiment and FDTD calculation for defect-free area. (a) Modal frequency shift vs $2 r / a$ characteristics. The plots show experimental results, whereas the dashed lines show calculated results. (b) Time-averaged $\left|H_{\mathrm{z}}\right|^{2}$ distribution for each mode for $2 r / a=0.62$. 